\title{
PERSPECTIVE
}

\section{Airway smooth muscle in asthma: flirting with disaster}

\author{
J.J. Fredberg
}

Airway hyperresponsiveness is the excessive narrowing of the airway lumen caused by stimuli that would cause little or no narrowing in the normal individual. It is one of the cardinal features of asthma but remains largely unexplained. Sometimes, though, clues to the greatest mysteries are right in front of us but we do not see them, and the role of breathing in airway hyperresponsiveness may be one of these clues.

The smooth muscle surrounding the airway shortens when it is activated, and as the muscle shortens the airway narrows and breathing tends to become difficult. The lung has a potent built-in defence against bronchospasm, however, and this defence works in the opposite direction; the act of breathing makes it difficult for activated muscle to shorten [1-8]. Asthma is an inflammatory disease, but could it be that it is the failure of this particular defence mechanism which is the most telling end-effect of the inflammatory cascade, and therefore the proximal cause of airway hyperresponsiveness in asthma? This idea is not at all new [1], but the emergence of new evidence and new understanding of underlying mechanisms invites reconsideration of this question.

We breathe all of the time and we sigh at the rate of about 10 times per hour [9]. The bronchodilating effect of this pattern of breathing is so effective that airway narrowing never approaches dangerous levels in healthy people, even when they fall into the hands of investigators who convince them to inhale high concentrations of bronchoconstricting agents. We can put the potency of this mechanism into perspective with the following observations. The expected physiological range of muscle stretch during spontaneous breathing is about $4-12 \%$ of muscle length whereas, in isolated activated muscle, tidal stretches of only $3 \%$ of muscle length are enough to inhibit active force generation by $50 \%$ [10]. By contrast, extremely high concentrations of isoproterenol $\left(>10^{-6} \mathrm{M}\right)$ are required to attain by purely pharmacological means the same degree of relaxation as is caused by the small tidal stretches which occur during breathing (unpublished observations). In healthy volunteers who inhale bronchoconstricting substances such as histamine, there is a reflex increase in the frequency and depth of spontaneous sighs when bronchospasm begins, and these deep inspirations cause prompt and nearly complete dilation of the airway $[4,11]$. Even when healthy volunteers inhale some of the most potent known bronchoconstrictors, such as leukotrienes, appreciable bronchospasm cannot be demonstrated unless deep

Correspondence: J.J. Fredberg, Dept of Environmental Health, Harvard School of Public Health, 665 Huntington Avenue, Boston, MA 02115 , USA. Fax: 16174323468.

Supported by PO1 HL33009 and RO1 HL 59682. inspirations are prohibited [12]. Taking into account the endogenous levels at which various dilators are found in the airway, these observations suggest that the tidal muscle stretches which are attendant to spontaneous breathing comprise the first line of defence against bronchospasm, and that tidal muscle stretch may be the most potent of all known bronchodilating agents.

In an asthmatic attack this bronchodilating mechanism fails. Indeed, there is ample evidence from the work of INGRAM and coworkers [11] to show that, if anything, in an asthmatic attack deep inspirations make matters worse. In this connection, experiments conducted years ago led FisH et al. [1] to the striking observation that airway obstruction in asthma behaves as if it were caused by an intrinsic impairment of the bronchodilating effect of a deep inspiration, as opposed to an inappropriate end-responsiveness of the airway itself. At about the same time, a similar observation led Овенек et al. [4] to speculate that asthma triggers a vicious circle in which asthmatic airway obstruction increases the frequency of deep inspirations, and deep inspirations, in turn, make the obstruction worse.

Although the underlying mechanism is unknown, the impairment of the bronchodilating effect of a deep inspiration is thought to be a feature characteristic of only the late-phase response to allergen challenge and of spontaneous asthmatic obstruction $[11,13]$. Therefore, it came as a surprise to learn only recently that an impairment of this kind is easily evoked in completely healthy individuals. Two laboratories have shown that if healthy, nonasthmatic, nonallergic subjects do nothing more than to refrain voluntarily from taking deep inspirations, within $15 \mathrm{~min}$ their airways become hyperresponsive to a degree that is virtually indistinguishable from that observed in asthmatic subjects [3, 14, 15]. Even more remarkably, when deep inspirations are eventually reinstated, the subsequent ability of deep inspirations to dilate the airways becomes profoundly impaired, just as it does in spontaneous asthmatic obstruction. Put simply, it is as if the airway smooth muscle, when activated, is all the time flirting with disaster and the mere removal of deep inspirations, which would seem superficially to be a rather trivial matter, is sufficient nonetheless to allow this flirtation to progress to a situation that is much more serious, even in healthy volunteers with no airway inflammation, no history of airway inflammation or allergy, and possessing airways and airway smooth muscle that are perfectly normal.

How is all this to be explained? Many mechanisms have been invoked, but perhaps the simplest may lie at the level of the cyclic interaction of myosin with actin, the molecular motor within the airway smooth muscle. When muscle contracts it also becomes stiff [10]. The muscle stiffens when activated because the binding of myosin to actin, 
which generates active force, also crosslinks the cytoskeletal matrix and these temporary links (bridges) turn over relatively slowly in time. Periodic muscle stretches caused by the action of tidal breathing have a direct mechanical effect at the level of this actomyosin interaction, however; they perturb this binding process and cause the bridges to detach much sooner than they otherwise would have, thereby decreasing the fraction of the time that myosin is attached to the actin filament. That is, tidal muscle stretches decrease the myosin duty cycle [16]. A reduced duty cycle implies fewer bridges attached at any moment and, accordingly, less muscle stiffness. As a result, the more the muscle stretches, the more compliant it becomes and, therefore, the easier it becomes to stretch. For the same reason, the more myosin binding is disrupted by the action of breathing, the more susceptible it becomes to further disruption. Muscle stretch acts as its own catalyst.

This describes positive feedback and a dynamic process that tends to be self-reinforcing. In this process, the tidal action of breathing creates force fluctuations that tend to bias myosin binding events and, due to this bias, the muscle equilibrates to a dynamically maintained length that systematically exceeds the length that would pertain with a time-invariant load of the same mean value; the muscle experiences systematic lengthening driven by force fluctuations. Taken together, these ideas comprise the perturbed equilibrium hypothesis [16], "perturbed" because the binding of myosin to actin is perturbed by the periodic muscle stretches associated with breathing and "equilibrium" because the muscle length (averaged over the tidal event) is set by a dynamically equilibrated steady state.

The good news is that when myosin binding is perturbed, the magnitude of the contractile response becomes functionally disengaged from the level of the contractile stimulus, much as stepping on the clutch pedal in a car disengages the wheels from the motor [16]. As a result, the muscle can remain compliant and extended even when the level of muscle stimulation is very high. Breathing is good for breathing. Or, putting it another way, if actomyosin is the molecular motor through which metabolic energy is converted into the mechanical energy that drives airway narrowing during bronchospasm, the tidal action of breathing works the other way round, using mechanical energy to disrupt those same molecular events and, thereby, hoist actomyosin on its own petard. Recent evidence suggests that it may be this dynamically equilibrated state of affairs that limits the extent of narrowing in the normal airway, and that the normal pattern of spontaneous breathing is sufficient to maintain it $[3,7,14-16]$, but only just.

The bad news is that this virtuous spiral of self-reinforcing events seems to be rather delicate and can unravel, or collapse on itself, if the amplitude of the force fluctuations that stretch the muscle should somehow become compromised. For example, force fluctuations are linked intimately to the peribronchial distending stress and its changes in time. Any factor that lessens peribronchial stress will decrease the force fluctuations, including thickening of the peribronchial adventitia, loss of lung elastic recoil, breathing at low lung volumes and failure to take deep breaths [17-21]. If so, then with each breath the muscle would stretch slightly less. If the muscle stretches less, then it will become stiffer still, and so on. Ultimately, the muscle can become so stiff that the physiological forces acting on the muscle become insufficient to stretch the muscle appreciably, leaving the muscle so stiff that it becomes virtually frozen and stuck at its static equilibrium length [16]. Bronchial hyperresponsiveness, the hypothesis suggests, might correspond to a failure of the underlying perturbed equilibrium to sustain itself, and an ensuing return of myosin to the binding equilibrium that exists in static conditions. Since the perturbed equilibrium of myosin binding inhibits shortening of activated muscle, the collapse of this dynamic system to static equilibrium conditions would represent the disinhibition of that process.

The perturbed equilibrium hypothesis has impressive explanatory power, as described below, but it requires us to shift our mindset about the nature of airway narrowing, not only quantitatively, but also qualitatively. The prevailing view holds that the airway is a statically equilibrated system in which airway calibre is set by the isometric steady-state force generated by the muscle, balanced by the passive reaction force developed by the load against which the muscle shortens [17-22]. But rather than being viewed simply as a balance of static forces, the perturbed equilibrium hypothesis implies that airway narrowing must be viewed in the context of the class of systems described by Nicolis and Prigogine [23]. The features of this class are conveniently illustrated in the familiar example of a spinning top: it is dynamic because it is spinning; it is conditionally stable because, in order to secure its stability and remain balanced upright on its point, it requires an ongoing flux of mechanical energy through the system; and stability and balance are emergent properties of the dynamic system rather than any one of its elements - if one observes a top that is not spinning, one cannot necessarily conclude that there is something wrong with the top. The perturbed equilibrium hypothesis suggests that the regulation of the airway lumen during muscle activation is, likewise, a conditionally stable dynamic process that is far away from static equilibrium conditions. With each breath the myosin binding is perturbed anew, keeping the process spinning, as it were, decreasing bridge numbers, increasing their rate of turnover and maintaining the system far from static equilibrium conditions, but only at a certain energetic expense. The hypothesis suggests, further, that destabilization of this dynamic process, and the resulting collapse to static binding equilibrium may be the primary mechanical consequence of thickening of the peribronchial adventitia that is associated with inflammatory remodelling of the airway in asthma, in which case force fluctuations acting on the muscle would be buffered and the muscle would then shorten, stiffen and find itself stuck in that state for as long as the muscle remains activated. If so, this frozen state would at long last provide a mechanism to explain the impairment in the ability of lung inflations to dilate the airway in asthma. In doing so, it would similarly explain the responses in healthy subjects challenged while deep inspirations are withheld and the subsequent impairment in the efficacy of deep inspirations, once reinstated, to dilate the airway. As discussed below, it would also explain hyperresponsiveness in subjects breathing at low lung volumes or with loss of lung recoil [19-21].

This picture may well be incorrect and it is almost certainly incomplete. Importantly, it does not take into account recent discoveries about smooth muscle plasticity that are bound to be important in understanding airway obstruction in asthma [24-26]. Nonetheless, the idea of 
perturbed equilibria of myosin binding seems to shed light on a variety of nagging puzzles that have surrounded airway reactivity and asthma. For example, when normal or asthmatic individuals are challenged with inhaled bronchoconstrictors in the standard fashion, the response of the asthmatic is characteristically different in two regards. In the asthmatic, the dose-response curve is shifted to the left, indicating hypersensitivity, but far more important than the shift is the increased magnitude of the response. With progressively increasing doses of bronchoconstrictor, the healthy subject displays a plateau of the response; at high agonist concentrations the airway is somehow protected and airway narrowing is limited. By contrast, in asthma the plateau is elevated, or no plateau can be established at all, in which case the protective mechanism fails and airway narrowing is limited only by airway closure $[21,27,28]$. P. Macklem, more than anyone, has made us aware that it is the mystery of this protective mechanism and how it fails that is the fundamental to understanding the pathophysiology of airway obstruction in asthma [18].

Based on the observation of an elevated or abolished plaeau response in asthma, investigators have reasoned, logically, that the muscle in the lungs of the asthmatic patient or the allergen-sensitized animal must be stronger or that the muscle load is reduced compared to healthy subjects (see below for more information on the latter). The former seemed the simplest explanation and was the oddson favourite, but what had seemed to be an almost certain outcome was not borne out in subsequent investigations of isolated muscle. The evidence is equivocal at best, but when available studies are taken together it seems that airway smooth muscle obtained at autopsy or from biopsies of living asthmatic patients produces no more isometric force than does normal muscle [29], while the isometric force-generating capacity is in any event more than sufficient to close all of the airways of the lung $[30,31]$. Yet, paradoxically, responses are limited in the normal individual but can become unlimited in asthma. The perturbed equilibrium hypothesis suggests a resolution of this paradox. It shows that abnormality of the muscle is not a precondition for abnormal muscle function. Inflammatory remodelling of the peribronchial connective tissues, for example, might be sufficient to destabilize the perturbed equilibria. Even with purely static muscle behaviour, reduced static load also implies reduced muscle length [21, 22]. However, should it collapse to a statically determined length, the muscle that had been maintained dynamically at a substantially elevated length (because it enjoyed the salutary effects of fluctuation-driven muscle lengthening) would have farther to fall. As such, the effect of connective tissue remodelling on muscle length might have two components, the dynamic component of which may be substantially amplified compared with the static component alone.

Even if the force-generating capacity of the muscle is normal, there may yet be a problem with airway smooth muscle in asthma after all. The problem, though, is not that the muscle is too strong, but rather that it is too fast! The perturbed equilibrium hypothesis leads naturally to the suggestion that fast muscle could be a problem because the faster the intrinsic rate of cycling of the myosin, the more difficult it is for breathing to perturb the reaction. That is, the faster the intrinsic rate of cycling, the faster a bridge, once becoming detached, will reattach and contrib- ute once again to active force and stiffness $[16,31]$. This facet of the hypothesis may help to explain the striking conundrum brought to our attention by STEPHENS and coworkers [32, 33], who have shown that circumstances in which bridge cycling rates are increased (reflected, for example, in an elevated speed of muscle shortening and elevated rate of utilization of adenosine triphosphate) have been consistently associated with airway hyperresponsiveness even though the isometric force generation capacity of the muscle is unchanged $[32,33]$. In practical terms, specific instances in which elevated rates of bridge cycling might come into play include the differences in airway responsiveness that have been observed between normal and allergen-sensitized muscle, between certain animal strains and, in some species, between mature and immature animals [34-36]. This rationale leads us, at long last, to the first plausible mechanism by which the rate of bridge cycling and its regulation may be reasonably thought to bear upon the prevalence of childhood asthma and its changes with lung maturation and allergic status.

The perturbed equilibrium hypothesis may tie together some other loose ends. Greater contractile response is predicted whenever the force fluctuations acting on the airway become compromised, not only when the peribronchial adventitia undergoes cytokine-driven inflammatory thickening [17, 19], but also when the lung loses elastic recoil or tidal lung expansion becomes diminished. These instances bring immediately to mind not only asthma, but also emphysema, normative ageing, restrictive disorders of the chest wall, obesity and spinal cord injury, each of which is known to be associated with a predisposition for airway hyperresponsiveness or asthma [37-42]. Similarly, a reduced amplitude of force fluctuations may come into play in nocturnal asthma [43]. Moreover, it is clear that when inflammatory remodelling of the airway does occur, the hypothesis predicts that the resulting predisposition for airway hyperresponsiveness might persist long after the inflammation itself is resolved [44, 45]. Finally, perturbed equilibria might also help to explain why the obstructive response in exercise-induced asthma typically begins only after cessation of the exercise, when tidal volumes have declined to resting levels.

While the ideas sketched above are of a speculative nature, they lead to three implications that will require further consideration. First, the old idea has been that airway smooth muscle length, and therefore airway calibre, is determined by a balance of two static forces: the isometric steady-state force generated by the muscle in balance with the passive reaction force developed by the load against which the muscle shortens. MACKLEM [46] has pointed out that once the muscle has become maximally stimulated, it is the forces and the loads that become all important, and that the level of the plateau response becomes essentially uncoupled from underlying biochemistry and cell biology. The static balance which MACKLEM [46] described remains highly relevant because it is the static state to which the dynamically equilibrated system would collapse if destabilized, but this picture is now seen to be incomplete. The view of the airway as a static mechanical system is now giving way to the more general idea of the airway as a system that is intrinsically dynamic, conditionally stable and far from static mechanical equilibrium. If this new picture is correct, then normal degrees of airway narrowing (normoreactivity) would have to be viewed in the context 
of a dynamic system that is tightly integrated across scales, with actomyosin reaction kinetics and cytoskeletal plasticity within the airway smooth muscle coupled directly to organ-level dynamic events such as tidal fluctuations in transpulmonary pressure, and vice versa. Accordingly, the system would be controlled by kinetic parameters, which depend on rate processes and time, not just static parameters, where rates and time are not factors. The pathobiology, i.e. collapse to static equilibrium conditions and excessive airway narrowing, would then be seen to be a consequence of the failure of that coupling. In that case, the muscle would shorten to a length dictated by static equilibrium conditions and remain frozen there, largely independent of respiratory events occurring at the organ level.

Recent advances in murine models have deepened our understanding of the immunological and genetic bases of airway hyperresponsiveness, and have shown that airway hyperresponsiveness can be uncoupled from airway inflammation [32, 47, 48], but we understand little of the specific mechanistic processes by which these factors lead to airway hyperresponsiveness and bronchospasm. Thus, the second implication is that this integrated point of view reinforces the notion that the inflammatory processes associated with asthma impact on more than the pharmacology of contractile agonists and the signal transduction cascade within the contractile apparatus. Rather, muscle stimulation is but one facet of a multifaceted inflammatory process that also causes cytokine-driven remodelling of airway connective tissues, alteration of airway smooth muscle mass, and modifications of the processes that regulate cross bridge cycling rates [17, 19, 32, 33, 45, 49]. These very factors may conspire to destabilize a conditionally stable, dynamic system that is far from static equilibrium, but is all the time flirting with disaster, like a spinning top that is wobbling but has not yet tumbled to rest. Should these factors change in concert, as they undoubtedly do in inflammatory airway disease, this might explain how rather small changes in each which, if taken alone, might seem inconsequential, when taken collectively might be sufficient to destabilize the process and, in doing so, precipitate a collapse to static conditions and a disproportionately large decrement of airway function.

Finally, the perturbed equilibrium hypothesis is attractive because it is rather simple yet seems to stitch together within a unified framework a diverse group of respiratory disease phenotypes that are largely unexplained and had been thought to be essentially unrelated. This integrated point of view suggests that as each of us focusses narrowly on the investigation of a particular mechanism to explain airway obstruction and airway hyperresponsiveness in asthma, we must bear in mind that all of these mechanisms may well make a contribution. In a system that is conditionally stable and precariously perched at the brink of destabilization, there may be no single cause responsible for its collapse, just as the last straw that breaks the camel's back is no more or less the cause than the first. During bronchospasm the airway lumen may be maintained by dynamic equilibrating mechanisms that are interconnected, comprising a web of causality, and every contributing factor may be important.

\section{References}

1. Fish JE, Ankin MG, Kelly JF, Peterman VI. Regulation of bronchomotor tone by lung inflation in asthmatic and nonasthmatic subjects. J Appl Physiol: Respir Environ Exercise Physiol 1981; 50: 1079-1086.

2. Green M, Mead J. Time dependence of flow-volume curves. J Appl Physiol 1974; 37: 793-797.

3. Skloot G, Permutt S, Togias A. Airway hyperresponsiveness in asthma: a problem of limited smooth muscle relaxation with inspiration. J Clin Invest 1995; 96: $2393-$ 2403.

4. Orehek J, Charpin D, Velardocchio JM, Grimaud C. Bronchomotor effect of bronchoconstriction-induced deep inspirations in asthmatics. Am Rev Respir Dis 1980; 121: 297-305.

5. Wheatley M, Pare PD, Engel LA. Reversibility of induced bronchoconstriction by deep inspiration in asthmatic and normal subjects. Eur Respir J 1989; 2: 331-339.

6. Violante B, Pellegrino R, Crimi E, Brusasco V. Increase in airway responsiveness and effect of deep inhalation on airway caliber in allergen-induced asthma. Am Rev Respir Dis 1992; 146: 127-131.

7. Shen X, Gunst SJ, Tepper RS. Effect of tidal volume and frequency on airway responsiveness in mechanically ventilated rabbits. J Appl Physiol 1997; 83: 1202-1208.

8. Nadel JA, Tierney DF. Effect of a previous deep inspiration on airway resistance in man. J Appl Physiol 1961; 16: 717-719.

9. Bendixen HH, Smith GM, Mead J. Pattern of ventilation in young adults. $J$ Appl Physiol 1964; 19: 195-198.

10. Fredberg JJ, Inouye D, Miller B, et al. Airway smooth muscle, tidal stretches, and dynamically determined contractile states. Am J Respir Crit Care Med 1997; 156: 1752-1759.

11. Lim TK, Pride NB, Ingram RH Jr. Effects of volume history during spontaneous and acutely induced air-flow obstruction in asthma. Am Rev Respir Dis 1987; 135: 591596.

12. Drazen JM, Austen KF. Leukotrienes and airway responses. Am Rev Respir Dis 1987; 136: 985-998.

13. Ingram RH Jr. Relationship among airway-parenchymal interactions, lung responsiveness, and inflammation in as-thma. Chest 1995; 107: 148S-152S.

14. King GG, Moore BJ, Pare PD. The time course of excessive airway narrowing caused by inhibition of deep inspirations during bronchoconstriction in normal subjects. Am J Respir Crit Med 1998; 157: A515.

15. Moore BJ, Verburgt LM, King GG, Pare PD. Effect of deep inspiration on methacholine dose-response curves in normal subjects. Am J Respir Crit Care Med 1997; 156: $1278-1281$.

16. Fredberg JJ, Inouye DS, Mijailovich SM, Butler JP. Perturbed equilibrium of myosin binding in airway smooth muscle and its implications in bronchospasm. $A m J$ Respir Crit Care Med 1998 (in press).

17. Macklem PT. A hypothesis linking bronchial hyperreactivity and airway inflammation: implications for therapy. Ann Allergy 1990; 64: 113-116.

18. Macklem PT. The mechanics of breathing. Am J Respir Crit Care Med 1998; 157: S88-S94.

19. Moreno R, Hogg JC, Paré PD. Mechanics of airway narrowing. Am Rev Respir Dis 1986; 133: 1171-1180.

20. Ding DJ, Martin JG, Macklem PT. Effects of lung volume on maximal methacholine-induced bronchoconstriction in normal humans. J Appl Physiol 1987; 62: 1324-1330.

21. Macklem PT. A theoretical analysis of the effect of airway smooth muscle load on airway narrowing. Am J Respir Crit Care Med 1996; 153: 83-89.

22. Lambert RK, Pare PD. Lung parenchymal shear modulus, 
airway wall remodeling, and bronchial hyperresponsiveness. J Appl Physiol 1997; 83: 140-147.

23. Nicolis G, Prigogine I. Exploring Complexity: An Introduction. New York, NY, WH Freeman \& Co., 1989.

24. Pratusevich VP, Seow CY, Ford LE. Plasticity in canine airway smooth muscle. J Gen Physiol 1995; 105: 73-94.

25. Gunst SJ, Meiss RA, Wu M-F, Rowe M. Mechanisms for the mechanical plasticity of tracheal smooth muscle. Am J Physiol 1995; 268: C1267-C1276.

26. Shen X, Wu MF, Tepper RS, Gunst SJ. Mechanisms for the mechanical response of airway smooth muscle to length oscillations. J Appl Physiol 1997; 81: 731-738.

27. Woolcock A, Salome CM, Yan K. The shape of the doseresponse curve to histamine in asthmatic and normal subjects. Am Rev Respir Dis 1984; 130: 71-75.

28. Sterk PJ, Bel EH. Bronchial hyperresponsiveness: the need to distinguish between hypersensitivity and excessive airway narrowing. Eur Respir J 1989; 2: 267-274.

29. Solway J, Fredberg JJ. Perhaps airway smooth muscle dysfunction does contribute to bronchial hyperresponsiveness after all. Am J Respir Cell Mol Biol 1997; 17: 144-146.

30. Warner DO, Gunst SJ. Limitation of maximal bronchoconstriction in living dogs. Am Rev Respir Dis 1992; 145: 553-560.

31. Gunst SJ. Contractile force of canine airway smooth muscle during cyclic length changes. J Appl Physiol: Respir Environ Exercise Physiol 1983; 53: 759-769.

32. Fan T, Yang M, Halayko A, Mohapatra SS, Stephens NL. Airway responsiveness in two inbred strains of mouse disparate in IgE and ILA production. Am J Respir Cell Mol Biol 1997; 17: 156-163.

33. Stephens NL, Laviolette M, Unruh H, Ma X. Contractility in bronchial airway smooth muscle cells obtained from asthmatic subjects by endobronchial biopsy is increased. Am J Respir Crit Care Med 1998; 157: A746.

34. Tepper B, Shen X, Bakan E, Gunst SJ. Maximal airway responses in mature and immature rabbits during tidal ventilation. J Appl Physiol 1995; 79: 1190-1198.

35. Mehta D, Shen X, Wu M-F, Tepper RS, Gunst SJ. The relationship between contractile protein activation and mechanical properties of mature and immature canine tracheal smooth muscle strips. Am J Respir Crit Care Med 1996; 151: A842.

36. Ikeda K, Mitchell RW, Guest KA, et al. Ontogeny of shor- tening velocity in porcine trachealis. Am J Physiol 262 (Lung Cell Mol Physiol 6) 1992; L280-L285.

37. Eden E, Mitchell D, Mehlman B, et al. Atopy, asthma and emphysema in patients with severe alpha-1-antitrypsin deficiency. Am J Respir Crit Care Med 1997; 156: 68-74.

38. Singas E, Lesser M, Spungen A, Bauman WA, Almenoff PL. Airway hyperresponsiveness to methacholine in subjects with spinal cord injury. Chest 1996; 110: 911-915.

39. Sparrow D, O'Connor GT, Rosner B, Weiss ST. Predictors of longitudinal change in methacholine airway responsiveness among middle-aged and older men: the Normative Aging Study. Am J Respir Crit Care Med 1994; 149: 376-381.

40. Boyer J, Amin N, Taddonio R, Dozor AJ. Evidence of airway obstruction in children with idiopathic scoliosis. Chest 1996; 109: 1532-1535.

41. Unger R, Kreeger L, Chistoffel KK. Childhood obesity. Medical and familial correlates and age of onset. Clin Pediatr 1990; 29: 368-373.

42. Camargo CA Jr, Weiss ST, Zhang S, Willett WC, Speizer FE. Prospective study of body mass index and risk of adult-onset asthma. Am J Respir Crit Care Med 1998; 157: A47.

43. Martin RJ. Nocturnal Asthma. Mount Kisco, Futura, 1993.

44. Crimi E, Spanevello A, Neri M, Ind PW, Rossi GA, Brusasco V. Dissociation between airway inflammation and airway hyperresponsiveness in allergic asthma. Am J Respir Crit Care Med 1998; 157: 4-9.

45. Haley KJ, Drazen JM. Inflammation and airway function in asthma - what you see is not necessarily what you get. Am J Respir Crit Care Med 1998; 157: 1-3.

46. Macklem PT. The clinical relevance of respiratory muscle research. Am Rev Respir Dis 1986; 134: 812-815.

47. De Sanctis GT, Merchant M, Beier DR, et al. Quantitative locus analysis of airway hyperresponsiveness in $\mathrm{A} / \mathrm{J}$ and C57BL/6J mice. Nature Genetics 1995; 11: 150-154.

48. Zuany-Amorim C, Ruffie C, Haile S, Vargaftig BB, Pereira P, Pretolani M. Requirement for gamma-delta T cells in allergic airway inflammation. Science 1998; 280: $1265-1257$.

49. Thomson RJ, Bramley AM, Schellenberg RR. Airway muscle stereology: implications for increased shortening in asthma. Am J Respir Crit Care Med 1996; 154: 749757. 